$\Rightarrow$ MALARIA

\title{
Sticking around
}

DOI:

10.1038/nrmicro1785
Although adults can develop clinical immunity to malaria, women who are pregnant for the first time are susceptible to infection with variants of Plasmodium falciparum that sequester in the placenta. Consequently, between 100,000 and 200,000 African infants die every year owing to pregnancyassociated malaria. However, women develop resistance to placental infected erythrocytes (IE) during successive pregnancies, which suggests that if the surface antigens on these erythrocytes could be identified it might be possible to develop a vaccine. Reporting in Infection and Immunity, Duffy and colleagues now show that the $P$. falciparum that causes pregnancy-associated malaria preferentially transcribes six genes, which could be important for vaccine development.

Research into the adherence of IEs to the placenta during pregnancyassociated malaria has focused on the var genes, which encode P. falciparum erythrocyte membrane protein 1 (PfEMP1; a family of variant proteins that are expressed on the erythrocyte surface), and specifically on var2csa, which seems to encode the dominant PfEMP1 that is associated with placental adhesion. However, evidence suggests that other, as yet unidentified, P. falciparum proteins are also required for the placental adhesion phenotype, possibly by contributing to the localization of PfEMP1. So, Duffy and colleagues set out to characterize this phenotype and identify any novel antigens that are involved.

Samples of $P$. falciparum from a small cohort of pregnant women were collected, and the whole-genome transcription profiles of these parasites were analysed and compared to the transcription profiles of P. falciparum parasites from children with malaria. As the P. falciparum gene-expression profile depends on cell-cycle timing, the authors also measured and made adjustments for the cell-cycle timing of the parasite to ensure they were comparing like with like. In addition to var2csa, five other genes - PFB0115w, PFD1140w, PF11785w, PFL0050c and MAL13P1.320 - were upregulated in parasites that caused pregnancyassociated malaria. Although the proteins encoded by these five genes are of unknown function, three contain putative export motifs, which suggests they could have a role in PfEMP1 localization.

Determining the functions of these genes and the mechanism by which they induce the placental adhesion phenotype could provide us with novel therapeutic targets.

Asher Mullard
ORIGINAL RESEARCH PAPER Francis, S. E. et al. Six genes are preferentially transcribed by the circulating and sequestered forms of Plasmodium falciparum parasites that infect pregnant women. Infect. Immun. 75, 4838-4850 (2007)

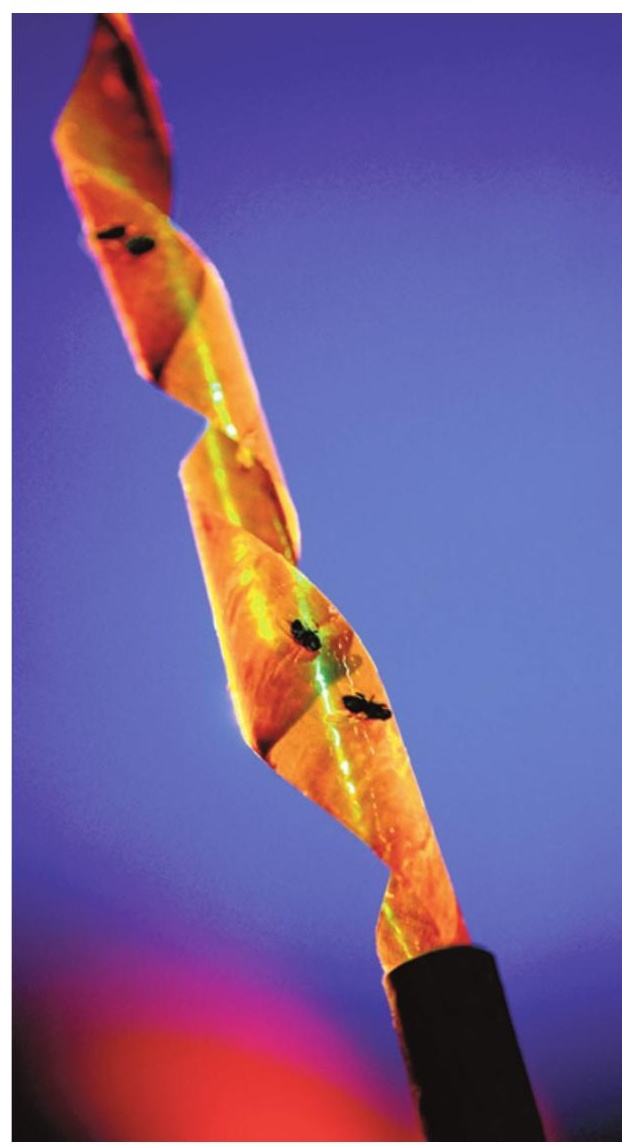

